\title{
Endoscopic Ultrasound-guided Thrombin Injection in Angiographically Unfeasible Giant Pseudoaneurysm of Gastroduodenal Artery in a Patient of Chronic Pancreatitis: An Unusual Case
}

\author{
Ashok Jhajharia, S. S. Sharma, Sandeep Nijhawan, Shekhar Puri, Kumar Shwetanshu Narayan, R. P. Singh, \\ Bhanwar Singh Dhandhu, Bharat Sapra
}

Department of

Gastroenterology, SMS

Medical College and

Hospital, Jaipur, Rajasthan, India

\begin{abstract}
Pseudoaneurysm is a known complication of pancreatitis associated with significant morbidity and mortality. Computed tomography (CT) localizes the pseudoaneurysm apart from the severity of underlying pancreatitis. Digital subtraction angiography with coil embolization is recommended treatment for pseudoaneurysm to avoid bleeding and surgery. However, in cases where angiographic coil embolization fails due to technical reasons, CT/ultrasonography- guided thrombin injection remains a viable option as described in literature. In the present case, role of endoscopic ultrasound-guided thrombin injection into large pseudoaneurysm that was not feasible by angiographic occlusion has been highlighted. The procedure avoided surgery and its related complication.
\end{abstract}

KEYWORDS: Chronic pancreatitis, pseudoaneurysm, thrombin

\section{INTRODUCTION}

P ancreatitis is associated with high morbidity Thortality. Pseudoaneurysm is most feared complication of chronic pancreatitis, noted in $10 \%$ of patients. The risk of rupture is as high as $50 \%$, and the mortality after rupture is about $15 \%-40 \%{ }^{[1,2]}$ Therefore, it requires a prompt diagnosis and management. Diagnostic modalities such as computed tomography (CT) help in picking up the lesion and delineating the vessel of origin. Digital subtraction angiography (DSA) and subsequent coil embolization are standard treatment protocol to manage such patients and avoid uncontrolled bleeding. Occasionally, coil embolization is not feasible due to inaccessible vascular territory or short neck of pseudoaneurysm. Further, some pseudoaneurysms may be angiographically occult and are seen on ultrasound or CT only. In such cases, ultrasound or CT guided percutaneous thrombin injection can be performed. Endoscopic ultrasound (EUS)-guided thrombin injection is a new development in this realm, especially in those patients where the visualization of the lesion is difficult on transabdominal ultrasound. This modality avoids the need for surgery and the associated morbidity, however, it requires expertise. We report a case where

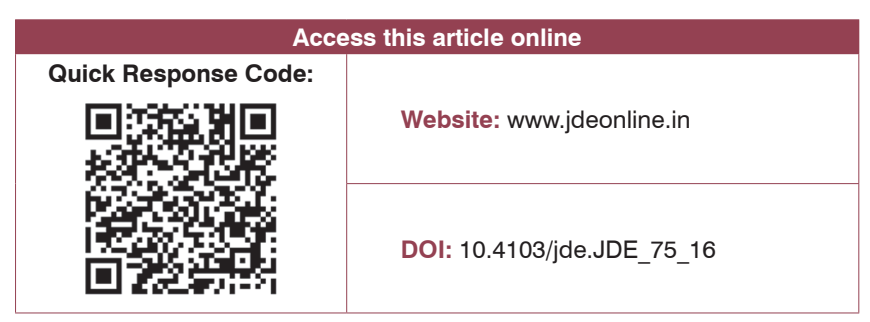

gastroduodenal artery (GDA) pseudoaneurysm were occluded using EUS-guided thrombin injection.

\section{Case Report}

A 43-year-old male presented to us with complaints of jaundice, pain abdomen, and shortness of breath. He was a chronic smoker and had history of significant ethanol intake. After evaluation, diagnosis of ethanol-induced chronic pancreatitis with complications in the form of walled-off pancreatic necrosis (WOPN), common bile duct (CBD) stricture, left-sided pleural effusion, and GDA pseudoaneurysm [Figure 1] was made. Patient underwent endoscopic retrograde cholangiopancreatography and a $7 \mathrm{Fr} \times 10 \mathrm{~cm}$ double pigtail stent was placed for CBD stricture to relieve jaundice. Following this, a surgical gastroenterology consultation was taken for operability, however, due to poor nutritional status (body mass index $<15 \mathrm{~kg} / \mathrm{m}^{2}$ ) and associated WOPN with pleural

Address for correspondence: Dr. Ashok Jhajharia, Department of Gastroenterology, SMS Medical College and Hospital, Jaipur, Rajasthan, India. E-mail: drashokjhajharia@gmail.com

This is an open access article distributed under the terms of the Creative Commons Attribution-NonCommercial-ShareAlike 3.0 License, which allows others to remix, tweak, and build upon the work non-commercially, as long as the author is credited and the new creations are licensed under the identical terms.

For reprints contact: reprints@medknow.com

How to cite this article: Jhajharia A, Sharma SS, Nijhawan S, Puri S, Narayan KS, Singh RP, et al. Endoscopic ultrasound-guided thrombin injection in angiographically unfeasible giant pseudoaneurysm of gastroduodenal artery in a patient of chronic pancreatitis: An unusual case. J Dig Endosc 2017;8:93-6. 
effusion, surgeon deferred surgery, and conservative management was advised. Following this, interventional radiology consultation was taken for embolization of GDA pseudoaneurysm, but it was deferred in view of involvement of a large caliber vessel which if embolized may cause dislodgement of foam particles leading to systemic embolization and serious complications. In our center, in hemodynamically stable patients, the usual approach to manage pancreatitis-related pseudoaneurysm is to perform CT angiography for localization of pseudoaneurysm. Subsequently, DSA is performed, and selective cannulation of the culprit vessel is done. If one can reach the pseudoaneurysm, coil embolization is done by occluding the back door, neck, and front door of the pseudoaneurysm. This prevents any collateral refilling of pseudoaneurysm. However, in our case, coil embolization could not be done due to above-mentioned reasons.

As this patient had pancreatic left pleural effusion, a possibility of pancreatic duct disruption in tail region was kept, and pancreatic duct stenting was planned. However, during pancreatic duct cannulation, there was a gush of blood from pancreatic orifice, most likely the aneurysm bleed, which was temporarily managed with mechanical compression using basket and local injection of epinephrine. We, therefore, planned EUS-guided thrombin injection to treat this pseudoaneurysm.

\section{Technique of endoscopic ultrasound-guided thrombin injection}

An experienced gastroenterologist, who has got experience in EUS-guided procedures, performed this procedure. The procedure was performed without any anesthesia. Initially, EUS was performed using curved linear array transducer (Olympus-GF-UCT180) in conjunction with EVIS EXERA CLV-180 light source (Olympus Medical System Corp., Tokyo, Japan), pseudoaneurysm was localized, and shortest path was chosen to target the pseudoaneurysm [Figure 2]. We used the thrombin component of Religare ${ }^{\circledR}$ Kit (Reliance), containing thrombin $500 \mathrm{IU} / \mathrm{ml}$. Before targeting the pseudoaneurysm, thrombin component of this kit was reconstituted with $1 \mathrm{ml}$ of calcium chloride, and this thrombin solution was further diluted with $6 \mathrm{ml}$ normal saline to a total of $7 \mathrm{ml}$ so that each milliliter of reconstituted solution contains approximate $71 \mathrm{IU}$ of thrombin. Further, each milliliter of reconstituted thrombin was transferred to $1 \mathrm{ml}$ insulin syringe, and 7 such thrombin-loaded syringes were kept ready before the procedure. Using 22-gauge Echo Tip Ultra EUS needle (Cook Medical Endoscopy, IN, United States), pseudoaneurysm was punctured under EUS guidance, and then, thrombin was injected in aliquots of $71 \mathrm{IU}$ till the pseudoaneurysm became echogenic resulting in thrombosis [Figure 3]. Under color Doppler, the success of thrombosis was confirmed, and the needle was

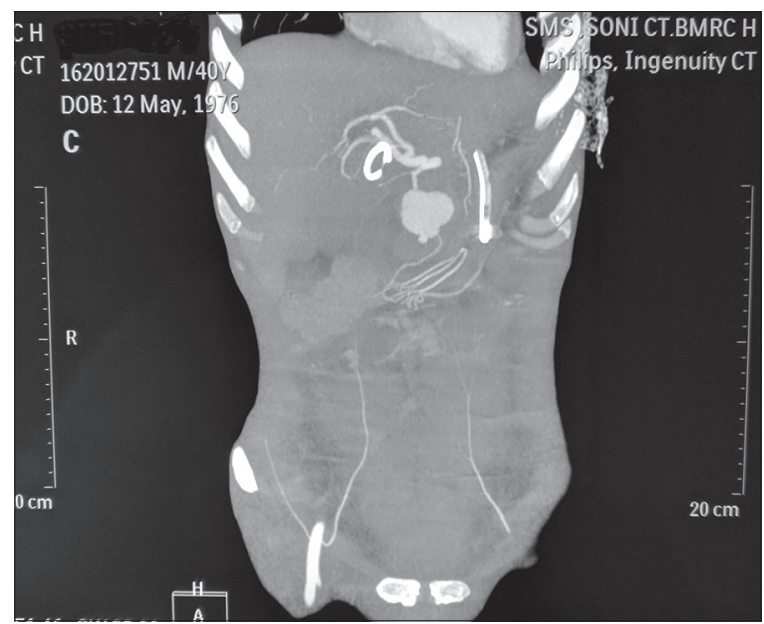

Figure 1: Gastroduodenal artery pseudoaneurysm on computed tomography angiography

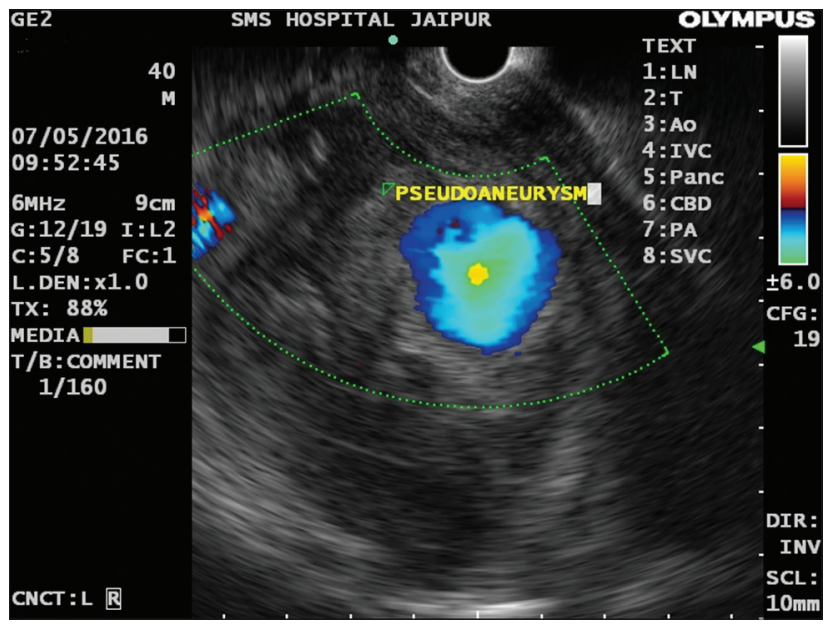

Figure 2: Endoscopic ultrasound shows pseudoaneurysm of gastroduodenal artery within walled of pancreatic necrosis with Doppler signal

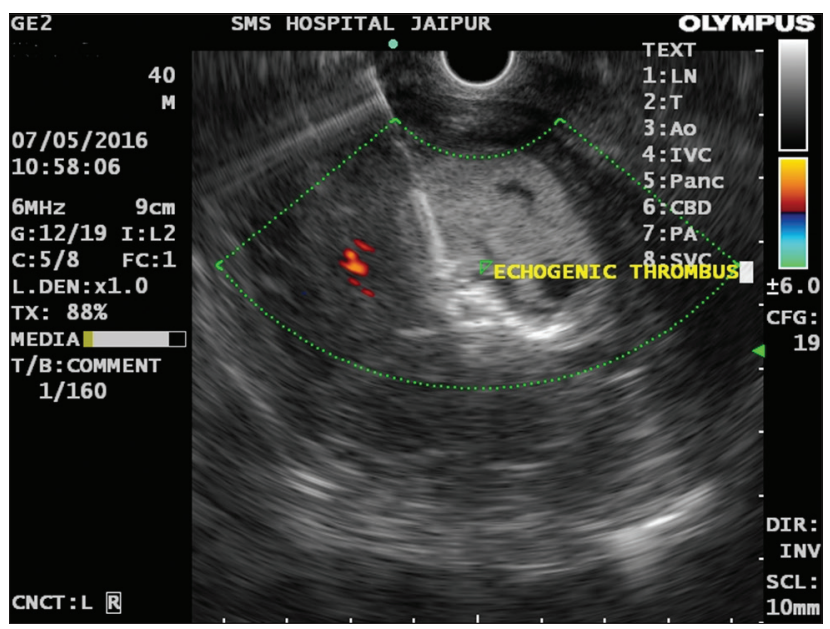

Figure 3: Endoscopic ultrasound needle 22-gauge-guided injection of thrombin with echogenic thrombus and loss of Doppler signal 
removed when loss of Doppler signal was seen inside the pseudoaneurysm sac. The entire procedure from start of localization of pseudoaneurysm to complete thrombosis of pseudoaneurysm lasted for about $15 \mathrm{~min}$. After procedure, the patient was monitored closely for signs of internal bleeding. Next day, check EUS was done that showed complete thrombosis of pseudoaneurysm. Follow-up after 10 days showed complete occlusion of the pseudoaneurysm [Figure 4].

Pancreatic duct was cannulated, and $7 \mathrm{Fr} \times 10 \mathrm{~cm}$ pancreatic stent was successfully placed. EUS again repeated at 3 months and showed no evidence of pseudoaneurysm [Figure 5].

\section{Discussion}

Pseudoaneurysm formation is a known vascular complication of pancreatitis. This occurs due to vessel injury as a result of proteolytic and lipolytic enzymes released during inflammation and pancreatic necrosis. ${ }^{[3]}$ The management of pseudoaneurysm in the setting of pancreatitis is challenging in view of the associated risk of rupture and hemodynamic compromise, apart from the increased morbidity of pancreatitis itself.

Diagnostic modality such as CT angiography remains a preliminary imaging modality in pancreatitis. DSA with coil embolization is the gold standard treatment. This offers the advantage of avoiding the associated surgical risk; besides, it ensures adequate thrombosis of the pseudoaneurysm in most cases. However, in situations where the access to pseudoaneurysm is not possible due to previous clipping/tortuous anatomy or when pseudoaneurysm is not visible on DSA due to slow filling and narrow neck; angiographic coil embolization may not be feasible. Some pseudoaneurysms are occult and detected only with other imaging modalities such as CT or EUS. If left alone, these pseudoaneurysms

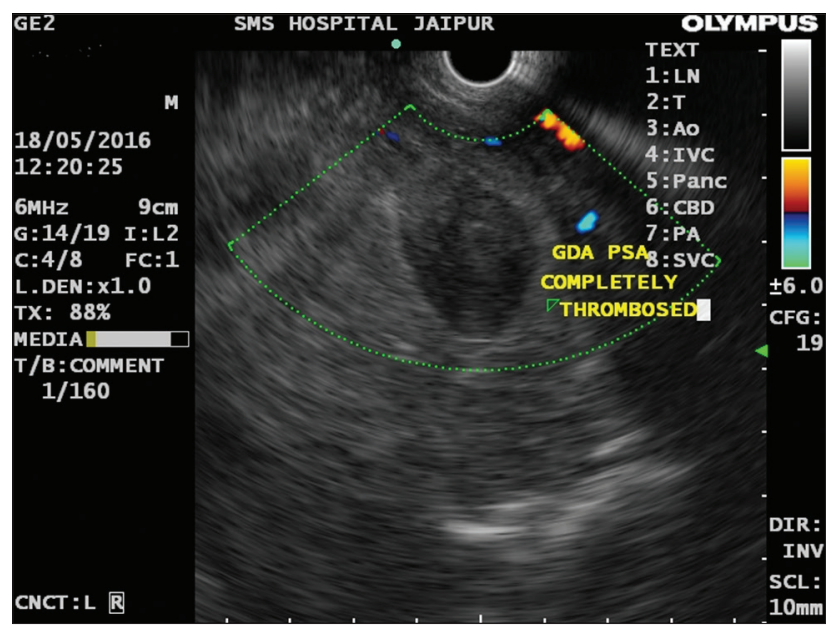

Figure 4: After 10 days, it shows no Doppler signal in pseudoaneurysm can rupture or rebleed. The risk of rupture in pancreatic pseudoaneurysms can been as high as $15 \%-40 \% .^{[1]}$ Hence, embolization is necessary to avoid rupture and rebleeding.

Thrombin remains an alternative to coils. It can be instilled directly at bleeding site, and flow cessation can be assessed. Thrombin is a good alternative in those cases, which are not feasible by endovascular route. The success of thrombin in embolization of peripheral pseudoaneurysms as $^{[4-6]}$ well as those of pancreas ${ }^{[7-10]}$ has been well described in literature. Thrombin can be given through transcutaneous route using ultrasound or CT guidance. However, in our patient, transabdominal ultrasound could not delineate the lesion clearly posing great challenge for thrombin instillation. EUS was the next step as most of these pseudoaneurysms are in the vascular territory in the vicinity of peripancreatic region. The advantage of EUS lies in clearly delineating the extent and size of pseudoaneurysm. Instillation of thrombin under EUS guidance requires a great deal of expertise. Thrombin injection ensures immediate occlusion of pseudoaneurysm in most of the cases. Response assessment can also be done easily. Hallmark finding is a complete loss of Doppler signal on EUS. Serial follow-up over 1 month did not reveal rebleeding or rupture in our case. There is scarcity of literature describing use of EUS-guided thrombin instillation in pancreatic pseudoaneurysm. In one of the earliest descriptions, Roach et al. ${ }^{[11]}$ described EUS-guided thrombin occlusion of a pseudoaneurysm arising from a branch of superior mesenteric artery in a patient presenting with the upper gastrointestinal bleed. This was done following failure to embolize angiographically as feeding artery could not be catheterized. Use of thrombin is recommended in cases of hemodynamically stable patients with small pseudoaneurysm. ${ }^{[12]}$ In addition, it can be used in

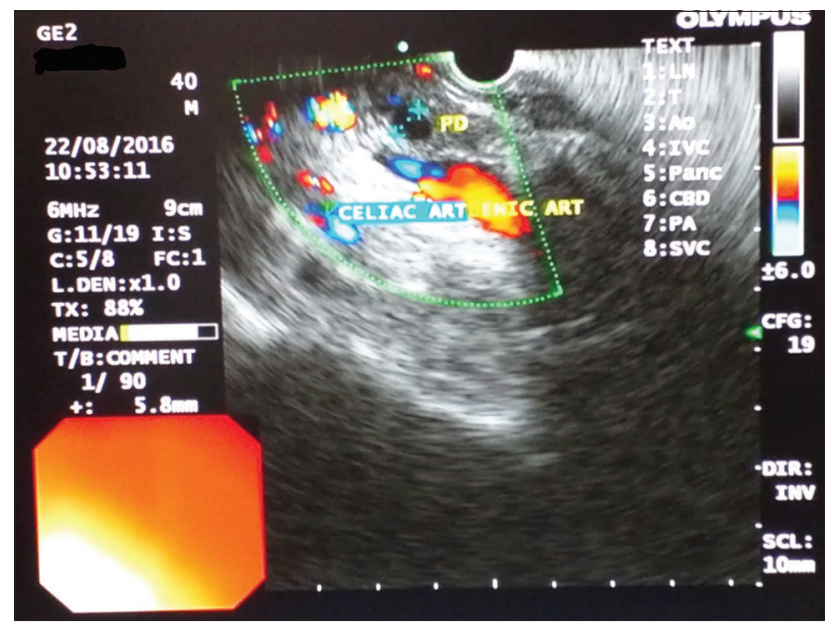

Figure 5: After 3 months, it shows no Doppler signal in pseudoaneurysm 
large lesions as an adjunct to coil embolization. In hemodynamically unstable patients and those with large pseudoaneurysms or those with failure after repeated embolization, surgery remains the only option. However, in the setting of pancreatitis, surgery is associated with a grim outcome. The advent of thrombin instillation through EUS guidance has opened an altogether new arena for managing difficult pseudoaneurysms and thereby avoiding associated surgical risk. This case was unique and possibly the first to the best of my knowledge in our country, where we used thrombin alone for a large pseudoaneurysm of $4 \mathrm{~cm} \times 5 \mathrm{~cm}$ size. Although one cannot undermine the expertise needed in EUS guidance, it offers new hope to this special group of patients with pseudoaneurysm, which are inaccessible to therapeutic angiography or through transabdominal ultrasound.

Limitations of thrombin instillation include the risk of distal thrombosis. This rarely occurs since thrombin is rapidly diluted and inactivated by the fast flowing blood stream. ${ }^{[10]}$ Immunological reactions such as hypersensitivity reactions are also known to occur. Recanalization after initial successful thrombosis of pseudoaneurysm is another complication. Finally, duration of follow-up required after treatment of pseudoaneurysms is not mentioned in the literature.

Further studies are also needed to provide data regarding efficacy and long-term outcomes.

\section{Financial support and sponsorship}

Nil.

\section{Conflicts of interest}

There are no conflicts of interest.

\section{REFERENCES}

1. Huizinga WK, Baker LW. Surgical intervention for regional complications of chronic pancreatitis. Int Surg 1993;78:315-9.

2. Gambiez LP, Ernst OJ, Merlier OA, Porte HL, Chambon JP, Quandalle PA. Arterial embolization for bleeding pseudocysts complicating chronic pancreatitis. Arch Surg 1997;132:1016-21.

3. Flati G, Andrén-Sandberg A, La Pinta M, Porowska B, Carboni M. Potentially fatal bleeding in acute pancreatitis: Pathophysiology, prevention, and treatment. Pancreas 2003;26:8-14.

4. Elford J, Burrell C, Freeman S, Roobottom C. Human thrombin injection for the percutaneous treatment of iatrogenic pseudoaneurysms. Cardiovasc Intervent Radiol 2002;25:115-8.

5. Friedman SG, Pellerito JS, Scher L, Faust G, Burke B, Safa T. Ultrasound-guided thrombin injection is the treatment of choice for femoral pseudoaneurysms. Arch Surg 2002;137:462-4.

6. Jaff MR. Pseudoaneurysms. Curr Treat Options Cardiovasc Med 2002;4:239-45.

7. Luchs SG, Antonacci VP, Reid SK, Pagan-Marin H. Vascular and interventional case of the day. Pancreatic head pseudoaneurysm treated with percutaneous thrombin injection. AJR Am J Roentgenol 1999;173:830, 833-4.

8. Francisco LE, Asunción LC, Antonio CA, Ricardo RC, Manuel RP, Caridad MH. Post-traumatic hepatic artery pseudoaneurysm treated with endovascular embolization and thrombin injection. World J Hepatol 2010;2:87-90.

9. Dambrin C, Marcheix B, Birsan T, Cron C, Muscari F, Suc B, et al. Posttraumatic pseudoaneurysm of the hepatic artery: Treatment with ultrasound-guided percutaneous transhepatic thrombin injection. J Trauma 2005;59:239-42.

10. Sparrow P, Asquith J, Chalmers N. Ultrasonic-guided percutaneous injection of pancreatic pseudoaneurysm with thrombin. Cardiovase Intervent Radiol 2003;26:312-5.

11. Roach H, Roberts SA, Salter R, Williams IM, Wood AM. Endoscopic ultrasound-guided thrombin injection for the treatment of pancreatic pseudoaneurysm. Endoscopy 2005;37:876-8.

12. Carr JA, Cho JS, Shepard AD, Nypaver TJ, Reddy DJ. Visceral pseudoaneurysms due to pancreatic pseudocysts: Rare but lethal complications of pancreatitis. J Vasc Surg 2000;32:722-30. 